\title{
A Photometric Investigation of a Bright Geminid
}

\author{
J. DEGEWI.J \\ Netherlands Association for Astronomy and Meteorology, \\ Zeiss Planetarium, Den Haag, Holland \\ AND \\ J. VAN DIGGELEN \\ University Observatory Sonnenborgh, Utrecht, Holland \\ Communicated by Zdeněk Kopal
}

Received July 14, 1967

\begin{abstract}
Photographic observations of meteors in the Netherlands started with a bright Geminid of photographic magnitude -8 observed on December $11,1955,21^{\mathrm{h}} 39^{\mathrm{m}} 55^{\mathrm{s}}$ by M. Alberts. From the assumed radiant and velocity we have constructed the trajectory of the bolide. The luminosity of the trail has been determined with a microphotometer. From the light curve we could derive the mass $(63 \mathrm{gm})$ and the diameter $(4 \mathrm{~cm})$ of the meteorite. Some traces of a wake of about $2^{\mathrm{m}}$ are visible in the interruptions of the trail.
\end{abstract}

\section{INTRODUCTION}

Photographic observations of meteors have been made in the Netherlands with eight small cameras distributed among some voluntary observers from 1950 . The first important result was a bright meteor photographed by $M$. Alberts in 1955, on December 11 at $21^{\mathrm{h}} 39^{\mathrm{m}} 55^{\mathrm{s}}$ UT. He worked at Alkmaar with a camera with a focal length of $140 \mathrm{~mm}$ and a focal ratio of $f / 2.8$, using Ilford HP3 plates $6 \times 9 \mathrm{~cm}$. The camera was equipped with a rotating shutter, which occulted the objective 25 times per second.

Unfavorable weather conditions prevented observations by other observers. $M$. Alberts, who photographed the bolide, saw it also visually as well as another observer in Aerdenhout.

A reproduction of the plate is given in Fig. 1. The meteor moved from left to right. Since the meteor was photographed from only one station, no direct calculations of height or velocity are possible. Reason- ably accurate heights may be obtained, however, by assuming a radiant position and velocity for the Geminid shower and then determining ranges and hence heights from the observed angular velocity.

The declination of the Geminid radiant was assumed at $\delta=32^{\circ} 44^{\prime}$ by interpolation from data by Lovell (1954) using the observed time of appearance. It was found that the observed trail passed through the declination of the apparent radiant if we assume that its right ascencion $\alpha=114^{\circ} 03^{\prime}$.

The trail of the Geminid on the plate is blurred by coma, so that the length of each of the segments is difficult to measure and we could not detect a marked deceleration along the trail. The angular velocity was assumed to be constant and to correspond to a meteoritic velocity of 35.95 $\mathrm{km} / \mathrm{sec}$. From the assumed radiant and velocity we have computed the heights along the trail. Errors in the assumed meteoritic velocity, radiant position, and speed of the shutter rotation, could result 


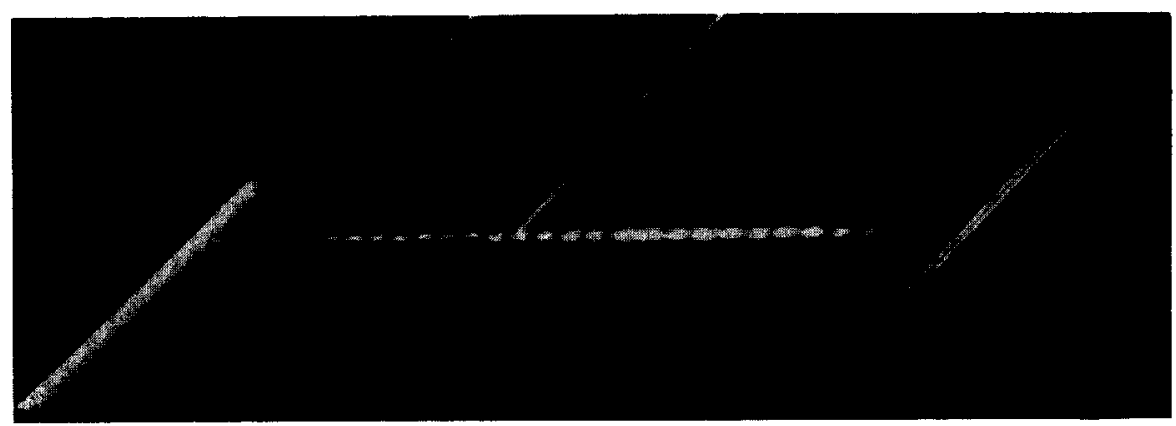

FIg. 1. Bolide photographed by M. Alberts; December 11, 1955, $21^{\mathrm{h}} 39^{\mathrm{m}} 55^{\mathrm{s}}$ UT.

in the heights being in error by as much as $5 \%$.

\section{The Photometry}

To estimate the luminosity, the image of the meteor was compared with star trails of spectral type AO, with the Utrecht microphotometer. The trail was measured two times: At first with a diaphragm limiting the luminous area on the plate to $0.06 \times 0.06 \mathrm{~mm}$ and with a luminous area of $3 \times 0.02 \mathrm{~mm}$. The second area was used for the brightest part of the trail, where the small area did not give a reliable result. Both series of measurements have been linked together with their overlapping parts. We also made a number of measurements of the trail perpendicular to its direction, and these have been compared with star trails measured in the same way. The luminosity of the measured points of the meteor trail was found from a curve (Fig. 2) in which the visual magnitudes of the stars were plotted against $(a b)^{x / 4}$, where $a$ is the peak density measured with the microphotometer and $b$ the half-width for $a=0.5$ of the measured density profile of the star trail. This method was introduced by Warner (1964). The meteor image trailed across the plate at a rate 2530 times greater than the image of the stars, corresponding to a difference of 8.5 magnitudes. This result may be influenced by such factors as emulsion sensitivity combined with the method of developing.

The luminosity of the measured points of the meteor trail have been reduced to the absolute luminosity at a distance of $100 \mathrm{~km}$ from the observer and corrected for atmospheric absorption. In this way we have found the luminosity curve of the Geminid (Fig. 3).

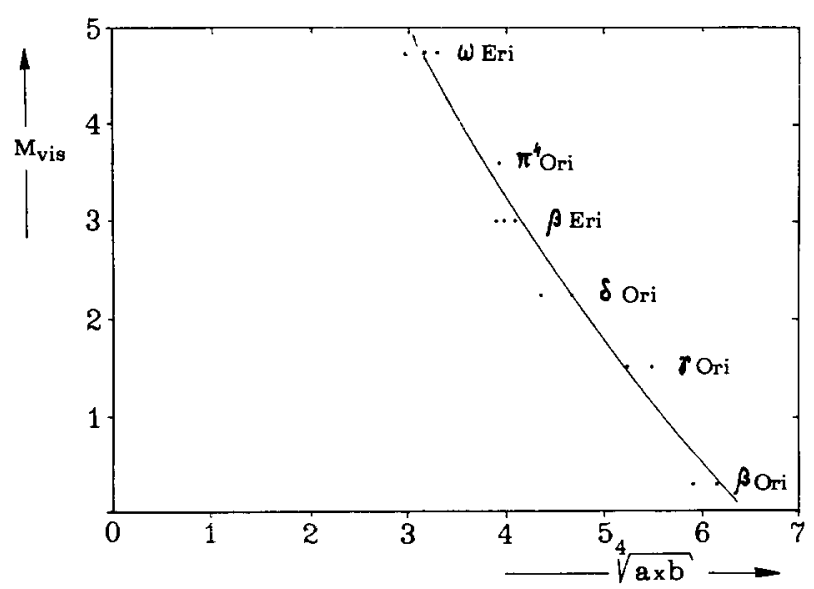

Fig. 2. Relation between $(a b)^{1 / 4}$ and the visual magnitude of the comparison stars. 


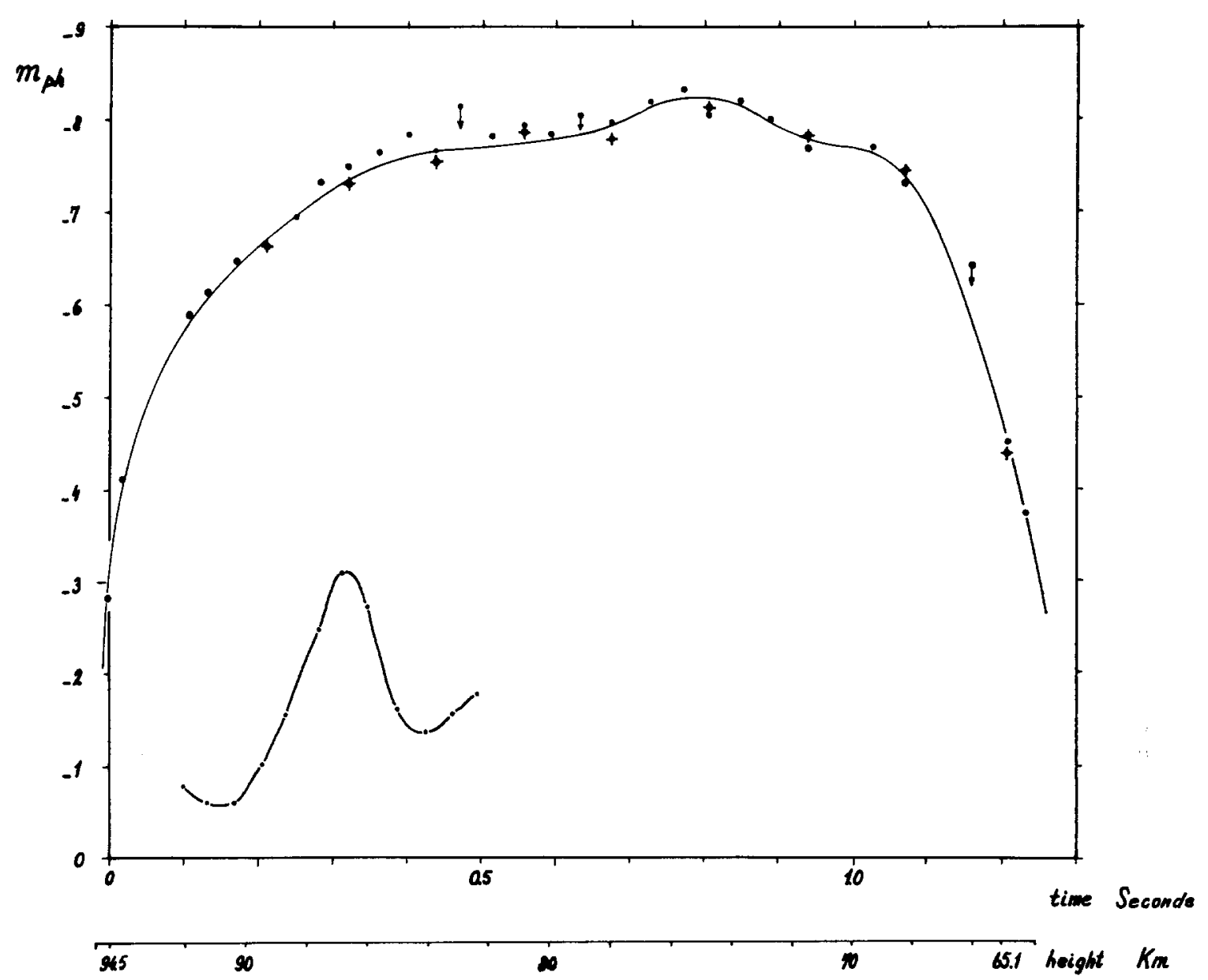

Fra. 3. Luminosity curve of the Geminid.

III. INDICATIONS OF THE EXISTENCE OF A WAKE

The photograph of this Geminid shows some indications of a luminous wake. As the objective of the camera, however, gives images affected with a strong coma, we cannot be certain that the faint images in the interruptions are not caused by scattered light from the bright trail. We have tried to prove the reality of the wake with two different methods.

With the Sabattier process we have made a series of reproductions of equidensity of the trail. Figure 4 shows clearly that the coma of the bolide is sharply bordered at a given level of intensity. This same isophote, however, has black regions in the interruptions which can only be caused

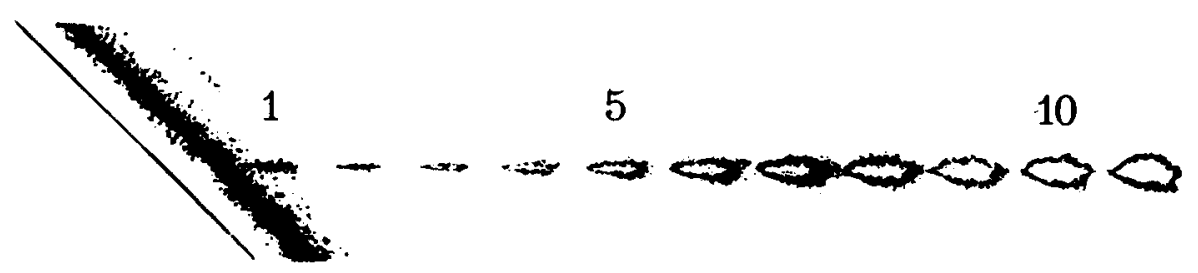

FIg. 4. Equidensity profiles of the meteor trail reproduced by the Sabattier process. 
by light of the wake. In the second place we have measured the luminosity profile of a star trail of a comparison star at about the same distance of the center of the plate as the wake trails. The luminosity profile of the streaks of the image of the bolide in the direction of its flight, cannot be directly compared with the star trail measured perpendicular to its direction. The interruptions are caused and the luminosity profile has been influenced by the rotation of the shutter. As we know the speed of the shutter, we can calculate the fraction of the objective covered during its passage and the theoretical diminishing of the brightness of the intensity profile given by the star trail by such a movement. At the same time the reduced theoretical profile must be shifted in accordance with the speed of the bolide. The method assumes that the emulsion does not give failures of the reciprocity law and that possible failure of reciprocity law effects are independent of the density.

From this calculation we found a theoretical profile showing the intensity along the border of the interruptions. A comparison of this curve with the observed one (Fig. 5) proves again the existence of the wake.

\section{Discussion of the Result}

We have not yet many exact observations of fireballs and only a very limited number of data on their brightness, light curve, and size. The accurate photographic reduction of this Geminid makes it possible to calculate these properties in detail. We have reduced the photographic light curve into a visual one with the aid of the color index $c_{i}=-1.8$ (Millman and Hoffleit, 1937). The absolute visual magnitude gives the intensity $I$ by the relation

$$
\log I=9.72-0.4 M_{v i s} .
$$

The mass of the bolide has been calculated from the formula

$$
m-m_{e}=2 \int \frac{I}{V^{2} \tau} \mathrm{d} t,
$$

where

$$
\begin{aligned}
\tau & =8.51 \times 10^{-10} \mathrm{~V} \\
V & =35.95 \times 10^{5} \mathrm{~cm} / \mathrm{sec},
\end{aligned}
$$

and we assume $m_{e}=0$. The result is that $m=63 \mathrm{gm}$. If we take the density of the meteorite $S=2.3$ according to the Geminids measured by Patashnick (1966) we

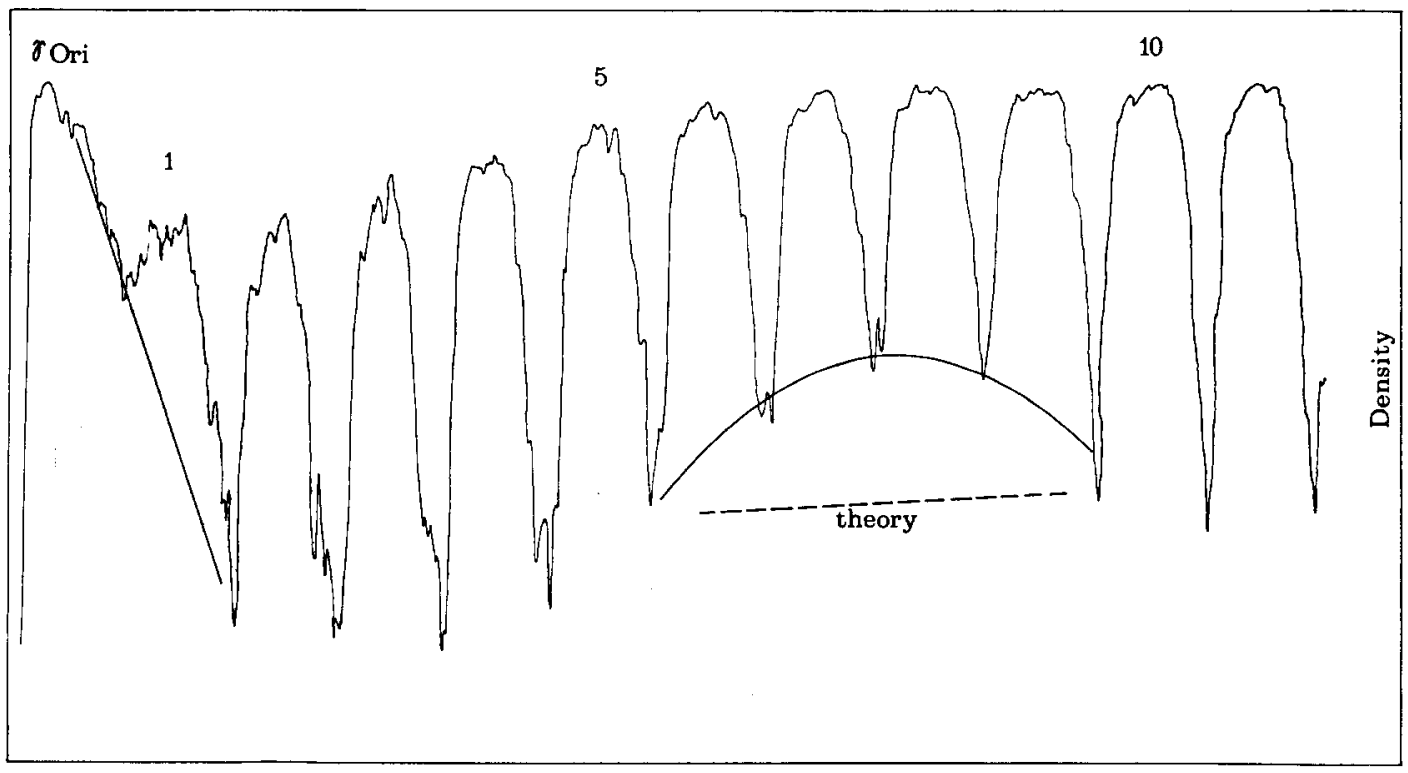

FIg. 5. Comparison of the theoretical intensity profile along meteor trail with the observed one. 


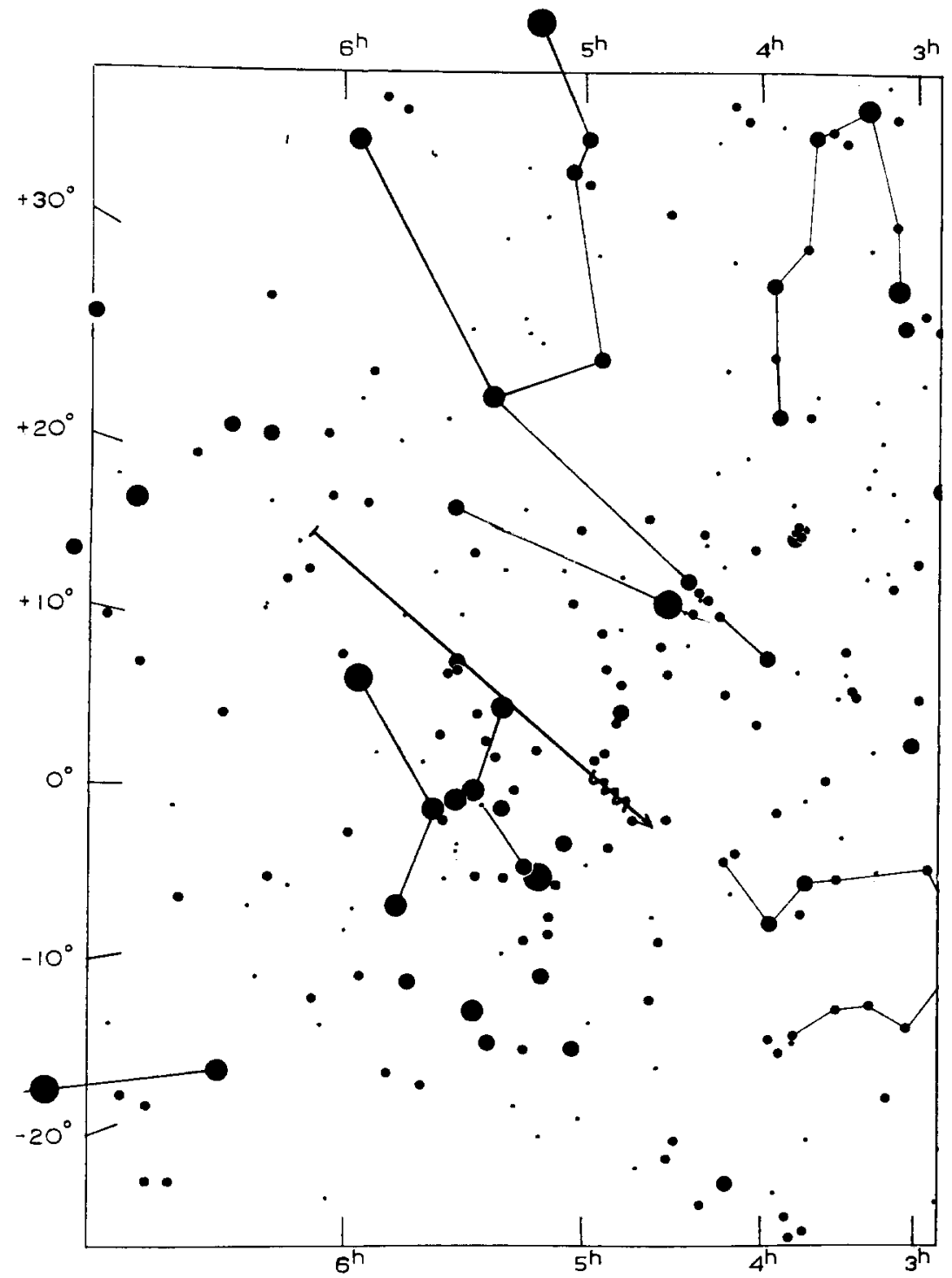

Fia. 6. Visual path of the bolide observed by M. Alberts.

find that the bolide may have been a sphere with a radius $r=1.86 \mathrm{~cm}$.

During his visual observations Alberts noticed the Geminid already at an earlier point. His visual path (Fig. 6) is divided into two clearly separated portions. The last part coincides with the photographic trail, the first part is not visible on the plate. It would be interesting to investigate why this happened, as such an effect has also been found in some other cases of bright meteors.
V. ACKNOWLEDGMen's

Many thanks for very valuable information and suggestions concerning the photometry are due to Mr. Th. van Dijk.

\section{REFERENCES}

Lovell, A. C. B. (1954). "Meteor Astronomy." Clarendon Press, Oxford, England.

Milliman, P. M., and Hoffleit, D. (1937). Ann. Astron. Obs. Harvard 105, 601.

Patashnick, H. (1966). Sky and Telescope 32, 207.

Warner, B. (1964). J. Brit. Astron. Assoc. 74, 99. 